\title{
A standardized description of European Sminthuridae (Collembola, Symphypleona), 2: review of four species of the genera Allacma and Spatulosminthurus
}

\author{
Pierre Nayrolles \\ Laboratoire de Zoologie, Ecobiologie des Arthropodes édaphiques, UPR CNRS 90 14, Université Paul \\ Sabatier, 118 route de Narbonne, F-31062 Toulouse Cédex, France
}

Keywords: Collembola, Symphypleona, Allacma, Spatulosminthurus, taxonomy, chaetotaxy, Europe

\begin{abstract}
According to our standard of the appendicular chaetotaxy, the following species are redescribed: Allacma fusca (Linné, 1758), Allacma gallica (Carl, 1899), Spatulosminthurus lesnei (Carl, 1899), and Spatulosminthurus betschi Nayrolles, 1990.
\end{abstract}

\section{Résumé}

Nous redécrivons, d'après la standardisation donnée pour la chétotaxie appendiculaire, les espèces suivantes: Allacma fusca (Linné, 1758), Allacma gallica (Carl, 1899), Spatulosminthurus lesnei (Carl, 1899) et Spatulosminthurus betschi Nayrolles, 1990.

\section{Introduction}

This is the second part of a series dealing with the European Sminthuridae. A standard of the appendicular chaetotaxy was proposed in my first paper ${ }^{1}$ (Nayrolles, 1993), and it will be used here without further explanations on the meaning of the symbols. All the species studied were collected in northeastern Spain and southwestern France. They belong to the genera Allacma and Spatulosminthurus.

Betsch \& Betsch-Pinot (1984) considered Allacma, Spatulosminthurus, and Sminthurus to form a monophyletic group based on the following putative apomorphies: postantennal seta with a very special morphology and ultrastructure (Altner et al., 1970), thick cuticle with a particular architecture, and well-developed tracheal system with chiasmata at the forelegs. Moreover, Betsch \& Waller (in press) point out the presence of a secondary (ontogenetic) neochaetosis on the great abdomen as a synapomorphy of these three genera (likely the consequence of an ontogenetic delay involving originally primary setae). The following putative evolved characters can be added: setae (AD)i0, (AD)i + 1, and (AD) i + 2 small and slender on a large base, and mucronal anterior lamella double.

I recall the abbreviations which correspond neither to setal symbols nor to the legend of the chaetotaxic tables; these were accurately explained in my first paper.

$$
\begin{aligned}
& \text { abd. = abdomen } \\
& \text { ad. = adult } \\
& \text { an. app. = anal appendage } \\
& \text { ant. = antennal segment } \\
& \text { ceph. diag. = cephalic diagonal } \\
& \text { juv. = juvenile } \\
& \text { oc = occurrence } \\
& \text { ov. org. = oval organ } \\
& \text { st. = instar }
\end{aligned}
$$

Allacma Börner, 1906

Some of the distinctively evolved characters of Allacma are: one pair of posterodorsal abdominal

\footnotetext{
${ }^{1}$ About this paper I correct a mistake that crept into the description of Lipothrix lubbocki: for the small abd. read: "Ratio an. app. : mucro = 1.1" instead of "Ratio mucro : an. app. = 1.1".
} 


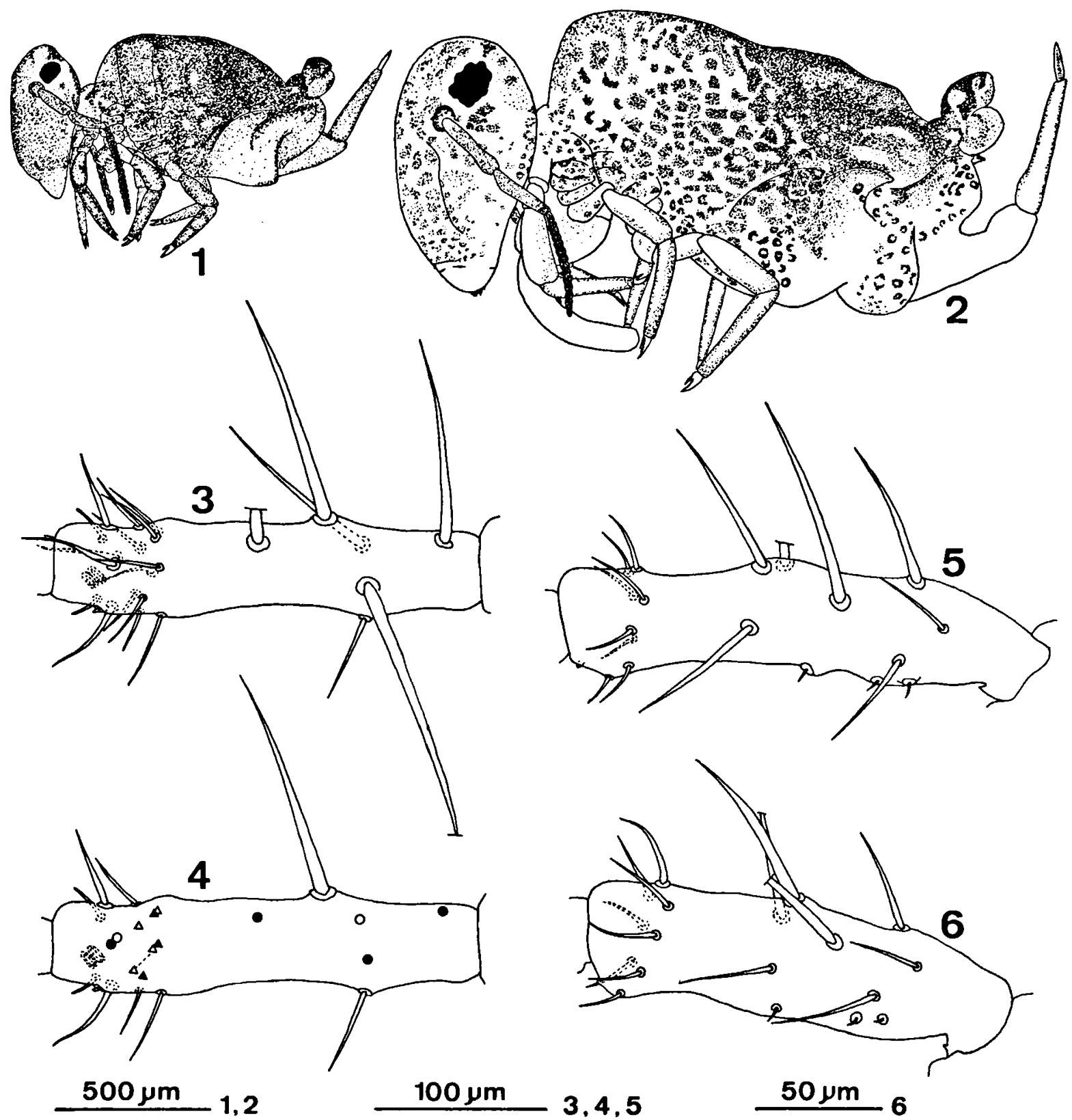

Figs. 1-5. Allacma fusca: 1 juv., habitus; 2, ad. female, habitus; 3, ad., ant. III, anterior view; 4, schematic representation of Fig. 3, setae of Ge and Gi drawn as those of antennal III organ (Xe, Xi) and its guard setae (Aai, Api, Ape), other setae are schematized as follows: a full symbol for a seta on reader's side (thus on anterior side), an empty symbol for a seta on opposite of reader's side, a circle for a primary seta, and a triangle for a secondary seta; secondary setae belonging to a same generatrix are linked together by a line which is continuous on the anterior side and discontinuous on the posterior side (in the case of $A$. fusca there is no continuous line because there are not two secondary setae on the anterior side belonging to the same generatrix); 5 , ad., ant. II, anterior view.

Fig. 6. Allacma gallica ad.: ant. II, anterior view.

glands from 2nd instar on, several setae of ant. II changing into macrochaetae, the reduced chaetotaxy of ant. III, and on this segment the dis- placement toward the apex of the secondary setae. Three species certainly belong to Allacma: $A$. fusca (Linné, 1758), A. gallica (Carl, 1899), and $A$. 
Table I. Appendicular chaetotaxy of Allacma fusca.

\begin{tabular}{|c|c|c|c|c|c|c|c|c|c|c|c|c|}
\hline \multirow{4}{*}{\begin{tabular}{l|l}
$A P$ \\
$A D$
\end{tabular}} & $\Delta$ & \multicolumn{11}{|c|}{ - } \\
\hline & $\bar{\Pi}$ & \multicolumn{11}{|c|}{$=$} \\
\hline & $\Delta$ & \multirow{2}{*}{\multicolumn{11}{|c|}{$\begin{array}{l}\text { D: io } \\
\text { io, it1 } \\
\text { Be, B }\end{array}$}} \\
\hline & & & & & & & & & & & & \\
\hline \multirow[t]{5}{*}{ AT } & $\Pi$ & \multicolumn{11}{|c|}{$=$} \\
\hline & \multirow[t]{4}{*}{$\Delta$} & & & $\mathbf{G e}_{\boldsymbol{\theta}}$ & Gae & Ga & Gai & $\mathbf{G}$ & Gpi & Gp & Gpe & \multirow{4}{*}{$\begin{array}{l}\text { Other setae: - } \\
\text { Be, Bae, Ba, Bai, and Bp become macrochaetae during } \\
\text { development. }\end{array}$} \\
\hline & & & Setae -1 & & & & $T$ & 0 & $T$ & $\mathbf{Q}$ & & \\
\hline & & & Setae 0 & $\mathbf{T}$ & D & D & & D & $\bar{D}$ & & D & \\
\hline & & & Setae +1 & & & & & & & & & \\
\hline \multirow[t]{6}{*}{ AQ } & str & \multicolumn{11}{|c|}{$\begin{array}{l}\text { B completely zoned. Section M euwhorled with } 11 \text { whorls. Right euwhorlation. } \\
\text { Subsegmentation formula: } 1+12+1=(A)+(M 1,12)+(B) \text { with BA more or less well individualized as a subsegment. } \\
\text { Right subsegmentation. }\end{array}$} \\
\hline & \multirow[t]{3}{*}{ A } & $\Pi$ & \multicolumn{10}{|c|}{ Allpi } \\
\hline & & $\mathrm{H}$ & \multicolumn{10}{|c|}{ Allleae, Allppe } \\
\hline & & $\bar{\Delta}$ & \multicolumn{10}{|c|}{$\begin{array}{l}\text { Apical bulb: joined to the apex } \\
\text { Allpe is bacilliform }\end{array}$} \\
\hline & \multirow[t]{2}{*}{$\begin{array}{l}\text { M } \\
\& \\
B\end{array}$} & $\mathbf{H}$ & \multicolumn{10}{|c|}{ 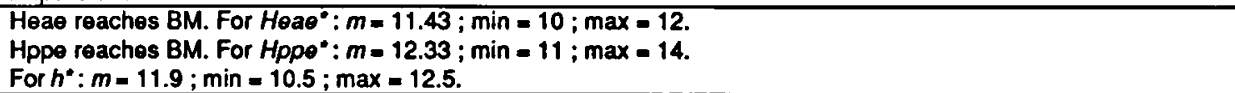 } \\
\hline & & $\mathbf{G}$ & \multicolumn{10}{|c|}{$\begin{array}{l}\text { Setae of BB: P: BBe, BBpe Q: BBae / BA complete whort / } n 80 \%(B M)-6-11 \text { / } n 80 \%(B)=17-21 / \text { all G } \\
\text { setae on } M \text { are present / n80\%(G) }=137-141 \text {. }\end{array}$} \\
\hline
\end{tabular}

\begin{tabular}{|c|c|c|c|c|}
\hline \multirow{2}{*}{ SB } & pre & $\overline{-}$ & + & + \\
\hline & $\Delta$ & P:1 sela & T:1 ov. org. & T:1 ov. org. \\
\hline$\widehat{S A}$ & $\Delta$ & - & P: 1 seta & P:1 seta \\
\hline$\overline{C X}$ & $\bar{\Delta}$ & $\overline{-}$ & 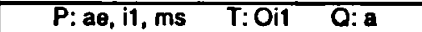 & $\begin{array}{lll}\text { Pa, i1, ms } & \text { T: a, Oi1 } & \text { Q: ai2 }\end{array}$ \\
\hline \multirow[t]{2}{*}{ TR } & $\bar{\Pi}$ & - & $\overline{-}$ & - \\
\hline & $\Delta$ & Q: pi & T: Oi1, Oi2 Q: ae, a2 & $\begin{array}{c}\text { T: a2, Oi1, Oi2 Q: ae } \\
p \text { is an acanthoid }\end{array}$ \\
\hline \multirow[t]{2}{*}{ FE } & $\Pi$ & - & - & $p \in 1$ \\
\hline & $\Delta$ & $\begin{array}{c}\text { delayed primordial seta: } \mathrm{Q}: \text { ae3 } \\
\begin{array}{cc}\mathrm{T}: \mathrm{pe3} & \mathrm{Q}: \mathrm{pi} 2, \mathrm{pe2}, \mathrm{Op}\end{array}\end{array}$ & $T: a 5$, ai2 $\quad Q: 22, a i 5, p e 3, O p$ & $\begin{array}{c}\mathrm{T}: \mathrm{a} 5, \mathrm{ai} 2 \\
\mathrm{Q}: \mathrm{a2}, \mathrm{ai3}, \mathrm{ai4}, \mathrm{ai5}, \mathrm{p} \text { 3, op }\end{array}$ \\
\hline \multirow[t]{4}{*}{$\mathrm{TT}$} & $\bar{V}$ & - & $V_{p}$ & $V_{p}$ \\
\hline & $\mathbf{K}$ & - & - & \multirow{2}{*}{$\frac{1}{+}$} \\
\hline & FP & \pm & + & \\
\hline & $\bar{\Delta}$ & $\begin{array}{l}\text { T: 4ai1, 4pi1, Vai, Vpi, FSa, O1pe, } \\
\text { O2pe, O3po, O4po } \\
\text { Q: } 2 a \theta, 3 a \theta, 3 a, 3 p, 4 a \theta 1,4 a 1,4 p 1, \\
\text { 4ai2, 4pi2, FSeT, FSai, FSpi, } \\
\text { FSpol, O1aө }\end{array}$ & $\begin{array}{l}\text { T: } 4 \text { ai1, 4i1, Vai, Vpi, FSa, O1pe, } \\
\text { O2pe, O3pe, O4pe } \\
\text { Q: 2ae, 3ae, 3a, 3p, 4ae1, 4a1, 4pi1, } \\
\text { 4ai2, 4pi2, FSeT, FSai, FSpi, } \\
\text { FSpe } \downarrow \text {, O1ae }\end{array}$ & $\begin{array}{l}\text { T: 3ai, 3i, 4ai1, 4i1, Vai, Vpi, FSa, } \\
\text { O1pe, O2pe, O3pe, O4pe } \\
\text { Q: 2ae, 2a, 2p, 3ae, 3a, 3pi, 3p, 4ae1, } \\
\text { 4a1, 4pi1, 4ai2, 4pi2, FS } \uparrow, \text { FSai, } \\
\text { FSpi, FSpe } \downarrow \text {, O1aө }\end{array}$ \\
\hline
\end{tabular}

\begin{tabular}{|c|c|c|c|c|c|c|c|c|c|c|c|c|}
\hline \multirow[t]{2}{*}{$M A$} & $\Pi$ & \multicolumn{11}{|c|}{ - } \\
\hline & $\Delta$ & \multicolumn{10}{|c|}{ T: a1, $p \in 2$} & \multirow{9}{*}{$\begin{array}{l}\text { Other setae: P:1p Q:4p, 5p, BBpi } \\
\text { Ba is a microchaeta. } \\
\text { IVp and Bp are bng setae becoming knobbed } \\
\text { during development. }\end{array}$} \\
\hline \multirow[t]{8}{*}{$\overline{D E}$} & $\Delta$ & \multirow{7}{*}{$\begin{array}{l}\text { Whorls } \\
\text { I to VI }\end{array}$} & & $\mathrm{Ge}_{\boldsymbol{\theta}}$ & Gae & $\mathrm{Ga}$ & Gai & Gi & Gpi & Gp & Gpe & \\
\hline & & & I & $\mathbf{P}$ & $\mathbf{P}$ & $\mathbf{P}$ & $\mathbf{P}$ & $\mathbf{P}$ & $\mathbf{P}$ & $\mathbf{P}$ & $\mathbf{P}$ & \\
\hline & & & II & & $\mathbf{P}$ & $\mathbf{P}$ & $\mathbf{P}$ & & $\mathbf{P}$ & $\mathbf{P}$ & $\mathbf{P}$ & \\
\hline & & & III & & $\overline{\mathbf{P}}$ & $\mathbf{P}$ & $\mathbf{P}$ & & $\mathbf{P}$ & $\mathbf{P}$ & $\mathbf{P}$ & \\
\hline & & & IV & & $\overline{\mathbf{P}}$ & $\mathbf{P}$ & & & $\bar{T}$ & $\bar{P}$ & $\mathbf{P}$ & \\
\hline & & & V & & & $\mathbf{P}$ & & & $T$ & $\mathbf{P}$ & $\bar{T}$ & \\
\hline & & & VI & & & & & & $\mathbf{Q}$ & 0 & $\mathbf{T}$ & \\
\hline & & \multicolumn{2}{|l|}{ WhorlB } & $\mathbf{T}$ & & $\mathbf{P}$ & & & $\overline{\mathbf{P}}$ & $\mathbf{P}$ & $\mathbf{P}$ & \\
\hline $\mathrm{MU}$ & $\bar{\Delta}$ & $\begin{array}{l}\text { Chaetotaxy: } \\
\text { lamella with a }\end{array}$ & & & & & & & & & & $\begin{array}{l}\text { broad at the basis, simple at the apex, outer } \\
\text { a serrated. }\end{array}$ \\
\hline
\end{tabular}


koreana (Yosii \& Lee, 1963). According to Yosii \& Lee (1963), Sminthurus purpurascens (Mac Gillivray, 1894) might be an Allacma as well. Living in Europe, $A$. fusca and $A$. gallica are the species I have studied.

Allacma fusca (Linné, 1758)

(Figs. 1-5 \& 7, Table I)

Material examined. - France, dép. Pyrénées Atlantiques, district Louvie-Juzon, locality Monguilholou, alt. $400 \mathrm{~m}$; forest of hazel tree, ash, and poplar, rather damp place with Geum urbanum L., Pulmonaria officinalis L., Helleborus foetidus L., Ligustrum vulgare L., Scolopendrium officinale Smith, Aspidium aculeatum Swartz, etc.; collected with a net by Nayrolles on unselected plants of the herbaceous stratum (station PA14c); 1-VIII-1987; 14 specimens; 3 ads. mounted.

France, dép. Pyrénées Atlantiques, district Mendive, between La Chapelle Saint-Sauveur and the Burdincurutcheta pass, alt. $960 \mathrm{~m}$; beech grove with spruces and some chestnut trees, many mosses on the ground, as well as Deschampsia flexuosa (L.), Luzula sp., Euphorbia amygdaloides L., Ilex aquifolium L., Pteridium aquilinum (L.), etc.; collected with a net by Nayrolles on unselected plants of the herbaceous stratum (station PA26a); 16-VIII-1987; 32 specimens; 11 juvs. and 6 ads. mounted.

Description. - Number of juv. st. $=4$.

Size ad. male: $1.7-2 \mathrm{~mm}$; female: $1.8-2.1 \mathrm{~mm}$. Color (Figs. 1-2). Color changes during development. Juv. with dark blue-purple pigment, especially on great abd. (dorsal area sometimes lighter); in ad. the pigment is less blue, with brown shades. Flanks and anterior area of great abd. with a lightening initially made up by small pale spots on a dark background. In old females the light patches sometimes blend and the dark pigment persists first as flecks and later as ocelli. Ocelli can also be present on head. Antennae purple, ant. IV being darker than the other antennal segments. Eyepatches black. Legs, and to a lesser extent furca, with purple pigment.

Great abd. Trichobothria: A, B, C appearing at 2nd st., in a very open inverted pattern. Dorsal setae: very rough macrochaetae. From 2 nd st. on, one pair of glands in posterodorsal area of great abd., with pores at cuticular level.

Antennae (Figs. 3-5). Antennal ratios: nearly the same for both sexes: ant. I : II : III : IV $=1$ : $2.5: 2.4: 4.2$ and ant. : ceph. diag. = 1.2. Chaeto- taxic variables: for $G: m=139.4 / s=2.16 /$ $\min =135 / \max =145$; for $I S h^{*}: m=0.91 / s=$ $0.036 / \min =0.81 / \max =0.96$; for $R S h: m=$ $0.74 / s=0.028 / \min =0.66 / \max =0.79$. Note: ant. II with 5 macrochaetae and 3 short and thin setae on large base, ant. III with 5 macrochaetae and few secondary setae shifted towards apex. Because of its reduced neochaetosis, the chaetotaxy of ant. III is rather difficult to interpret and its understanding is based upon comparisons with other genera.

Head. Eyes: $8+8$; eyepatch with 2 setae. Frons and vertex with rough macrochaetae. One pair of postantennal setae rounded and finely serrated at apex. Labral formula: 6/5-5-4.

Legs. (CX1)il absent. Femur: cup present. Tibiotarsal inner side with spine-like thick rough setae. Claws of all legs with a large tunica and large pseudonychia. Tunica and pseudonychia grow during development. All legs without teeth on inner crest of claw. Outer lamella of empodial appendage without teeth on fore- and mesolegs, but with a small tooth on hindlegs. Empodial filament mediumsized (nearly half length of empodial corpus) and rather thick at basis.

Ventral tube. Sacs warty from 2 nd st. on. Chaetotaxy: apical flaps with one pair of primary setae; one pair of setae on corpus appearing on 4th st.

Retinaculum. Rami bidentate from 1st st. on (apical tooth distinct and joined with median tooth at 1 st st., and becoming indistinct during development). Chaetotaxy: on anterior lobe one pair of setae appears at 3rd st., another pair at 4th st.

Furcula (Fig. 7). Several noteworthy characters on dentes: Vae and VIa absent, IVp and Bp knobbed, and $\mathrm{Ba}$ changed into microchaeta.

Small abd. Made up of abd. V + abd. VI. Trichobothrium: D present. Genital papilla of male medium-sized with about 30 setae. An. app. long and serrated at apex. Ratio an. app. : mucro $=0.9$.

Allacma gallica (Carl, 1899)

(Figs. 6, 8, 9, Table II)

Material examined. - France, dép. Ariège, district Bédeilhac-etAynat, between Bédeilhac and Saurat, alt. $620 \mathrm{~m}$; near a stream 

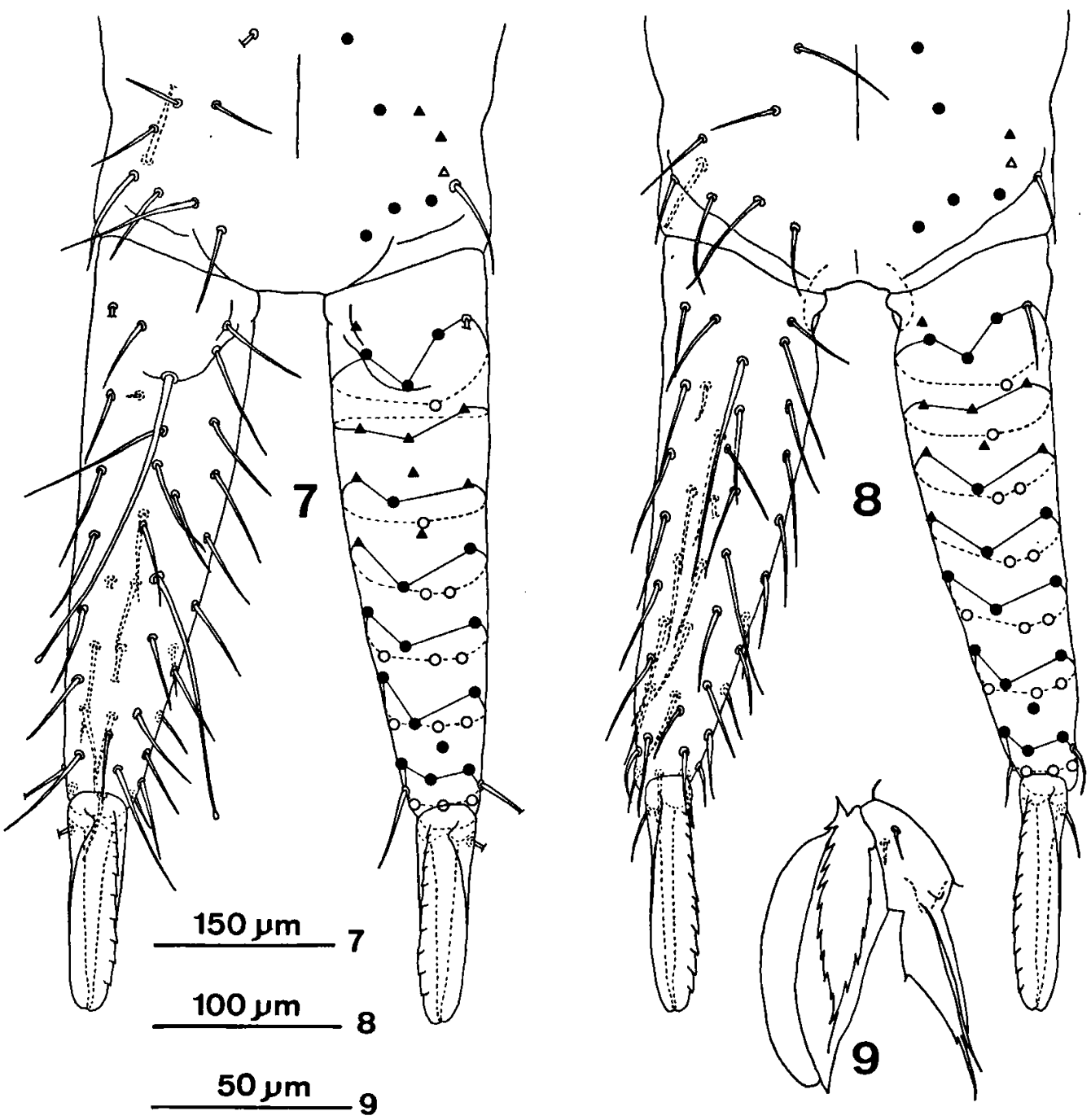

Fig. 7. Allacma fusca ad.: furcula, posterior view; on the right, schematic representation as follows: setae of Ge and Gi drawn, a full symbol for a seta on reader's side (thus on posterior side), an empty symbol for a seta on opposite of reader's side, a circle for a primary seta, and a triangle for a secondary seta; setae belonging to a same whorl are linked together by a line which is continuous on the posterior side and discontinuous on the anterior side.

Figs. 8-9. Allacma gallica ad.: 8, furcula, posterior view; on the right, same schematic representation as in Fig. 7; 9, hind pretarsus, anterior view.

under trees, with: Urtica dioica L., Symphytum tuberosum L., Pulmonaria officinalis L., Anemone nemorosa L., Galium aparine L., etc.; collected with a net by Nayrolles on unselected plants of the herbaceous stratum (station AR12b); 15-IV-1988; 4 juvs.; 2 mounted.

France, dép. Landes, district Samadet, road D2, near the river Le Gabas, alt. $70 \mathrm{~m}$; shady place, with Fraxinus excelsior L., Sorbus torminalis Crantz, Corylus avellana L., Quercus patraea Lieblen, Brachypodium pinnatum Palisot de Beauvois, Brachypodium silvaticum Roemer \& Schultes, Molinia caerulea (L.),
Carex sp., etc.; collected with a net by Nayrolles on unselected plants of the herbaceous stratum (station LA4); 22-X-1987; 14 specimens; 6 juvs. and $4 \% \varnothing$ mounted.

France, dép. Lot-et-Garonne, district Moncaut, near the stream Le Petit Auvignon, alt. $70 \mathrm{~m}$; wood with Pinus silvestris L. and Quercus pubescens Willdenow, and in undergrowth: Juniperus communis L., Hedera helix L., Cornus sanguinea L., Rubia peregrina L., etc.; collected with a net by Nayrolles on unselected plants of the herbaceous stratum (station LG1); 10-X-1989; 8 specimens; 3 juvs. and 3 ads. mounted. 
Table II. Appendicular chaetotaxy of Allacma gallica.

\begin{tabular}{|c|c|c|c|c|c|c|c|c|c|c|c|c|}
\hline \multirow{4}{*}{ AP } & $\Delta$ & \multicolumn{11}{|c|}{ i is a microchaeta } \\
\hline & $\Pi$ & \multicolumn{11}{|c|}{-} \\
\hline & $\Delta$ & \multirow{2}{*}{\multicolumn{11}{|c|}{$\begin{array}{l}\text { D: io } T: 0+1, i+1 \quad Q: a+1, i+2 \\
\text { io, } i+1, \text { and } i+2 \text { are small and slender setae on large base. } \\
\text { Bae and Bpo become macrochaetae during development. } 0+1 \text { is also a macrochaeta. }\end{array}$}} \\
\hline & & & & & & & & & & & & \\
\hline \multirow[t]{5}{*}{ AT } & $\Pi$ & \multicolumn{11}{|c|}{$=$} \\
\hline & $\Delta$ & & & Go & Gae & $\mathbf{G a}$ & Gai & $\mathbf{G}$ & Gpi & $G p$ & $G p$ & \multirow{4}{*}{$\begin{array}{l}\text { Other setae: - } \\
\text { Be, Bae, Ba, Bai, and Bp bocome macrochaetae during } \\
\text { development. }\end{array}$} \\
\hline & & & Setae -1 & & & & $\mathbf{T}$ & $\bar{Q}$ & $T$ & $\mathrm{Q}$ & & \\
\hline & & & Setae 0 & $\mathbf{a}$ & $\bar{D}$ & D & & D & $\overline{\mathrm{D}}$ & & $\bar{D}$ & \\
\hline & & & Setae +1 & & & & & & & & & \\
\hline \multirow[t]{6}{*}{$\overline{A Q}$} & str & \multicolumn{11}{|c|}{$\begin{array}{l}\text { B completely zoned. Soction M euwhorled with } 10 \text { or } 11 \text { whorls. Right ouwhorlation. } \\
\text { Subsegmentation formula: } 1+10 / 11+1=(A)+(M 1,10 / 11)+(B) \text {. Right subsegmentation. }\end{array}$} \\
\hline & \multirow[t]{3}{*}{$\mathbf{A}$} & $\Pi$ & \multicolumn{10}{|c|}{ Allpi } \\
\hline & & $\bar{H}$ & \multicolumn{10}{|c|}{ Allleae, Allippe } \\
\hline & & $\bar{\Delta}$ & \multicolumn{10}{|c|}{$\begin{array}{l}\text { Apical bulb: joined to the apex } \\
\text { Allpe is bacilliform }\end{array}$} \\
\hline & \multirow[t]{2}{*}{$\begin{array}{c}\text { M } \\
\& \\
B\end{array}$} & $\bar{H}$ & \multicolumn{10}{|c|}{$\begin{array}{l}\text { Heae reaches BA. For } \mathrm{Heas}^{\circ}: m=11.29 ; \min =10 ; \max =12 \text {. } \\
\text { Hppe reaches BM. For Hppo }: m=12.13 ; \min =11 ; \max =13 \text {. } \\
\text { For } h^{\circ}: m=11.7 ; \min =11.0 ; \max =12.5 \text {. }\end{array}$} \\
\hline & & $\mathbf{G}$ & \multicolumn{10}{|c|}{$\begin{array}{l}\text { Setae of BB: P: BBe, BBpe / BA with several variable setae / n80\%(BM) =3-8/ } \mathrm{n} 80 \%(B)=11-19 / \mathrm{G} \text { setae } \\
\text { on } \mathrm{M} \text { are rarely lacking } / \mathrm{n80} \%(G)=120-128 \text {. }\end{array}$} \\
\hline
\end{tabular}

\begin{tabular}{|c|c|c|c|c|}
\hline \multirow{2}{*}{5} & pre & - & + & + \\
\hline & $\Delta$ & P: 1 seta & \multirow{2}{*}{$\frac{\text { P: } 1 \text { seta Q: } 1 \text { ov. org. }}{P: 1 \text { sela }}$} & $\mathrm{T}: 1$ ov. org. \\
\hline SA & $\Delta$ & - & & P: 1 seta \\
\hline $\mathrm{CX}$ & $\bar{\Delta}$ & P:i1 & P: ae, i1, ms $\quad$ T:Oi1 $\quad$ Q: a & P:ae, i1, ms $\quad$ T: a, Oi1 $\quad$ Q: ai2 \\
\hline \multirow[t]{2}{*}{$\pi R$} & $\bar{\Pi}$ & $=$ & - & - \\
\hline & $\Delta$ & Q: pi & T: Oi1, Oi2 Q: ae, a2 & $\begin{array}{c}\text { T: Oi1, Oi2 Q: ae, a2 } \\
p \text { is an acanthoid }\end{array}$ \\
\hline \multirow[t]{2}{*}{ FE } & $\Pi$ & - & - & pe1 \\
\hline & $\bar{\Delta}$ & $\begin{array}{l}\text { delayed primordial seta: } \bar{Q}: a \theta 3 \\
\text { T:pe3 } \quad \text { Q: pi2, pe2, Op }\end{array}$ & T: a5, ai2 Q: a2, ai5, po3, Op & $\begin{array}{c}\mathrm{T}: \mathrm{a5}, \mathrm{ai} 2 \\
\mathrm{Q}: \mathrm{a2}, \mathrm{ai3}, \mathrm{ai4}, \mathrm{ai5}, \mathrm{pe3}, \mathrm{Op}\end{array}$ \\
\hline \multirow[t]{4}{*}{$\pi$} & $\mathbf{v}$ & $=$ & $\overline{V_{p}}$ & $V_{p}$ \\
\hline & $\bar{K}$ & - & \multirow{2}{*}{$\frac{-}{+}$} & \multirow{2}{*}{$\frac{-}{+}$} \\
\hline & FP & + & & \\
\hline & $\Delta$ & $\begin{array}{l}\text { T: } 4 \text { ai1, 4pi1, Vai, Vpi, FSa, O1po, } \\
\text { O2pe, O3pe, O4pe } \\
\text { O: } 2 \mathrm{ae}, 3 \mathrm{ae}, 3 \mathrm{a}, 3 p, 4 a \theta 1,4 a 1,4 p 1, \\
\text { 4ai2, 4pi2, FSeT, FSai, FSpi. } \\
\text { FSpel, O1ae }\end{array}$ & $\begin{array}{l}\text { T: } 4 \text { ai1, 41, Vai, Vpi, FSa, O1pe, } \\
\text { O2pe, O3pe, O4pe } \\
\text { Q: 2ae, 3ae, 3a, 3p, 4ae1, 4a1, 4pi1, } \\
\text { 4p1, 4ai2, 4pi2, FSe } \uparrow, \text { FSai, FSpi, } \\
\text { FSpe } \downarrow \text {, O1ae }\end{array}$ & $\begin{array}{l}\text { T: 3ai, 3i, 4ai1, 4i1, Vai, Vpi, FSa, } \\
\text { O1pe, O2pe, O3pe, O4pe } \\
\text { Q: } 2 a e, 2 a, 2 p, 3 a e, 3 a, 3 p i, 3 p, 4 a e 1, \text {, } \\
\text { 4a1, 4pi1, 4p1, 4ai2, 4pi2, FSe } \uparrow \text {, } \\
\text { FSai, FSpi, FSpe } \downarrow \text {, O1 ae }\end{array}$ \\
\hline
\end{tabular}

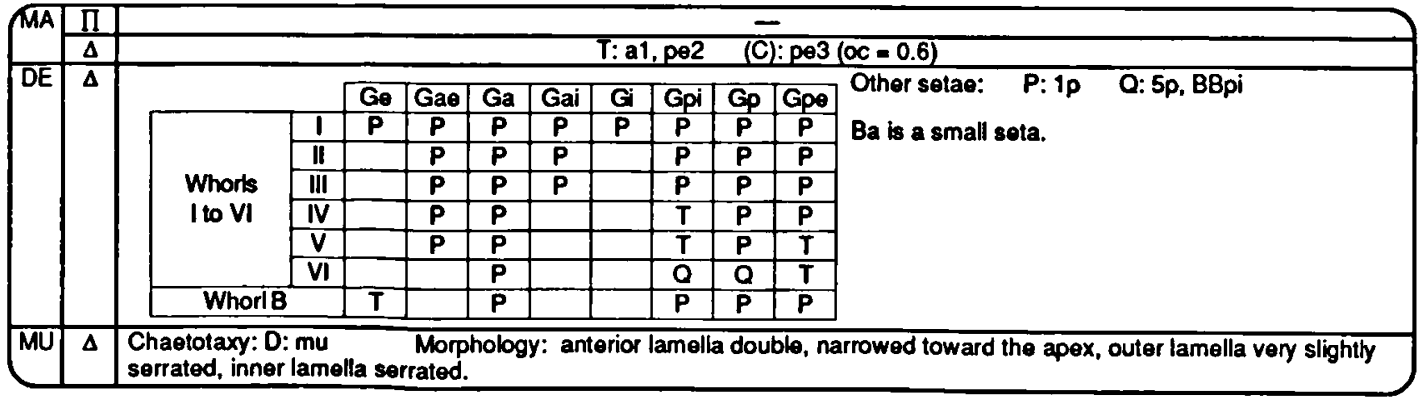


France, dép. Gironde, district Naujac-sur-Mer, locality Lizan, alt. $15 \mathrm{~m}$; wood of maritime pine and birch with Molinia caerulea (L.), Erica cinerea L., Erica ciliaris L., Osmunda regalis L., Ulex nanus Smith, etc.; collected with a net by Nayrolles on unselected plants of the herbaceous stratum (station GI5); 23-X-1987; 20 specimens; 4 juvs. and 4 ads. mounted.

\section{Description. - Number of juv. st. $=4$.}

Size ad. male: $1.2-1.3 \mathrm{~mm}$; female: $1.3-2 \mathrm{~mm}$. Color. Background color of body generally very dark blue-purple, almost black, back of great abd. sometimes without pigment. Head black-purple dorsally behind eyes, and light, often white or sometimes brown, elsewhere. Frons with three small black-purple patches linked together, one pair at internal area of the antennal insertion and the unpaired spot on frontal eye.

Great abd. Trichobothria: A, B, C appearing at 2nd st., in a very open inverted pattern. Dorsal setae: rough macrochaetae sharply ending in spike. One pair of prominent glands in posterodorsal area of great abd.

Antennae (Fig. 6). Antennal ratios: in female, ant. I : II : III : IV = $1: 2.2: 2.6: 5.6$ and ant. : ceph. diag. = 1.6; in male, ant. I : II : III : IV = $1: 2.3: 2.7: 6.3$ and ant. $:$ ceph. diag. $=1.7$. Chaetotaxic variables: for $G: m=124.1 / s=2.89$ $/ \min =118 / \max =129 ;$ for $I S h^{*}: m=1.00 / s$ $=0.036 / \min =0.96 / \max =1.09$; for $R S h$ : $m=0.82 / s=0.023 / \min =0.78 / \max =0.85$. On ant. II, in comparison with $A$. fusca, note the following differences: $\mathrm{Be}$ is rather thick but with medium length (so it is not considered as a macrochaeta), a 1 medium-sized and not broadened. For $a+1$ the difference from $A$. fusca is very sharp. As in $A$. fusca, the understanding of the chaetotaxy of ant. III is based upon comparisons with other genera.

Head. Eyes: 8+8; eyepatch with 2 setae. Interocular area and vertex with large rough macrochaetae. One pair of postantennal setae pointed and finely serrated at apex. Labral formula: 6/5-5-4.

Legs (Fig. 9). Femur: cup present. Tibiotarsal inner side with spine-like thick rough setae. Claws of all legs with a large tunica and large pseudonychia. Tunica and pseudonychia grow during development. All legs without teeth on inner crest of claw. Outer lamella of empodial appendage without teeth on fore- and mesolegs, but with a small tooth on hindlegs. Empodial filament medium-sized (nearly half length of empodial corpus) and rather thick at basis.

Ventral tube. Sacs warty from 3rd st. on (sacs not observed on 2nd st.). Chaetotaxy: apical flaps with one pair of primary setae, corpus with a pair from 4th st. on.

Retinaculum. At 1st st. rami tridentate, bidentate from 2nd. Chaetotaxy: on anterior lobe one pair of setae appears at 3rd st., another pair at 4th.

Furcula (Fig. 8). Note Gp with only two setae (1p and $5 p$ ) in interwhorls.

Small abd. Made up of abd. V + abd. VI. Trichobothrium: D present. Genital papilla of male medium-sized with about 30 setae. An. app. rather long and distally serrated; outer edge finely serrated beyond half its length, the serrated section on inner edge being little shorter. Ratio an. app.: mucro = 0.7 .

Discussion. - The dental chaetotaxy is the most useful criterion for distinguishing both species of Allac$m a$ : absence of (DE)Vae and (DE)VIa, and presence of long and knobbed setae on $\mathrm{Gp}$ in $A$. fusca, conversely (DE)Vae and (DE)VIa are present in $A$. galli$c a$ and no knobbed seta is present on the posterior (dorsal) side of the dens. These differences have been noted by Ellis (1973).

\section{Spatulosminthurus Betsch \& Betsch-Pinot, 1984}

In 1984, Betsch \& Betsch-Pinot isolated some species of Sminthurus into the new genus Spatulosminthurus. Although this distinction is justified, it is nevertheless true that the Sminthurus - Spatulosminthurus group is monophyletic, with Allacma as sister group. As the synapomorphies characterizing the monophyletic group Sminthurus - Spatulosminthurus we can note: (TR3)p is not an acanthoid but a mesochaeta (this is a reversion), (AQ)AIIp is a blunt microchaeta (remark that in Allacma this seta has already undergone a slight size decrease since its form is not crook-like as usually but bacilliform), and the length of ant. IV (with notably $m(G)$ over 150).

As remarked by Betsch \& Betsch-Pinot (1984), 

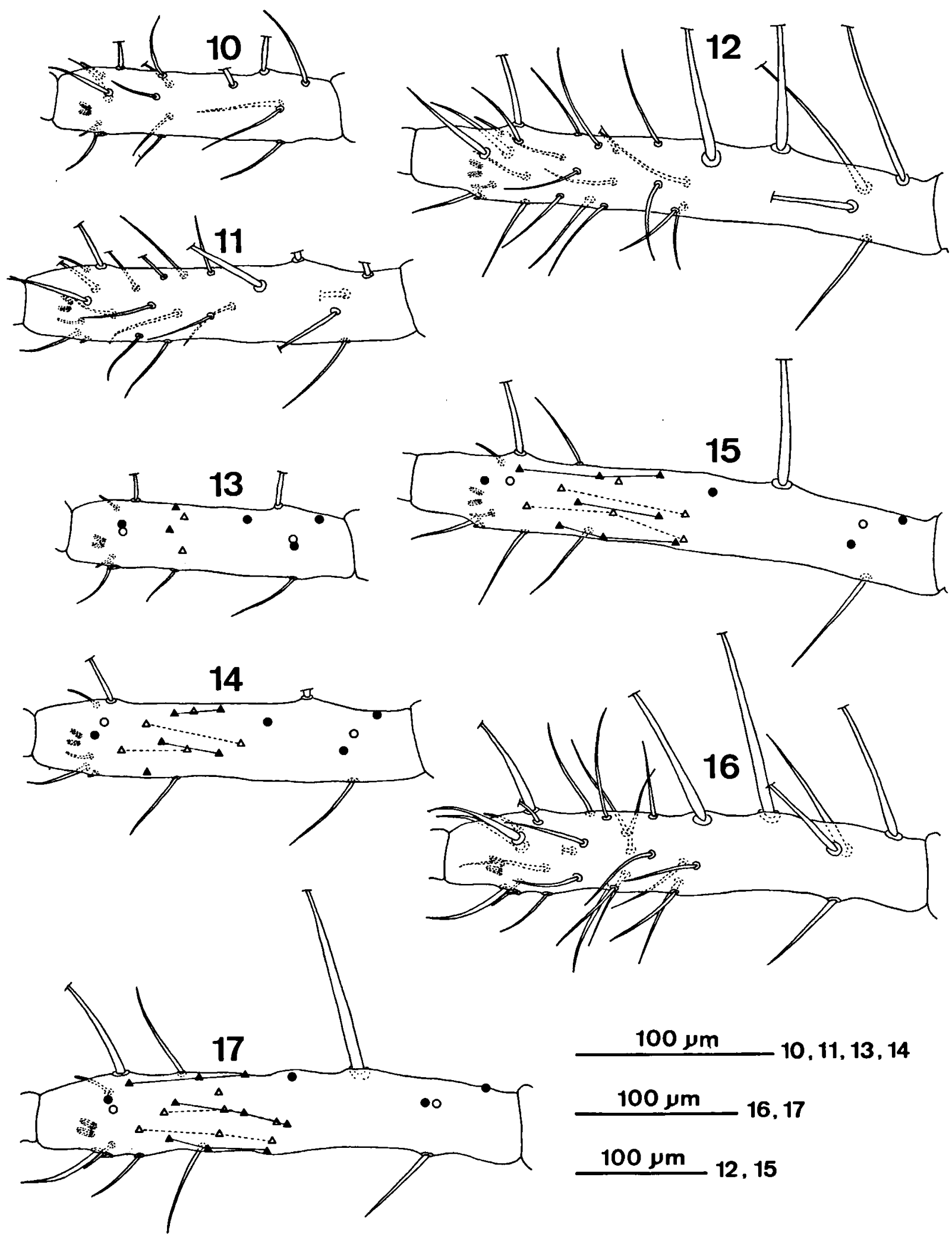
Spatulosminthurus has many autapomorphies, for example: spatulate setae at apex of tibiotarsi, (TI.)Vi changed into microchaeta, female circumanal setae broadened, and short and thick an. app.

Spatulosminthurus includes six species: gattoi (Stach, 1967), flaviceps (Tullberg, 1871), bulgaricus (Rusek, 1965), guthriei (Stach, 1919), lesnei (Carl, 1899), and betschi Nayrolles, 1990. I collected only $S$. lesnei and $S$. betschi, so herein I give a redescription of lesnei and a complement to my description of betschi.

\section{Spatulosminthurus lesnei (Carl, 1899)}

(Figs. 10-15, Table III)

Material examined. - Spain, prov. Barcelona, between Vallgorguina and Arenys de Munt, alt. $300 \mathrm{~m}$; long grass with Dactylis glomerata L., Pteridium aquilinum L., Origanum vulgare L., etc.; collected with a net on unselected plants by Nayrolles (station BA15a); 4-IX-1987; 6 juvs.; 5 mounted.

Same place; on a track in undergrowth, various gramineae and Agrimonia eupatoria L., Geum urbanum L., Betonica officinalis L., Plantago major L., etc.; collected with a net on unselected plants by Nayrolles (station BA15b); 4-IX-1987; 5 juvs.; 1 mounted.

Spain, prov. Gerona, near Vilajuïga, alt. $70 \mathrm{~m}$; garrigue with Lavandula stœchas L., Thymus vulgaris L., Rosmarinus officinalis L., Ruta sp., etc.; collected with a net on unselected plants by Nayrolles (station GE3a); 25-IV-1987; 3 ads.; 2 mounted.

Spain, prov. Gerona, between Lloret de Mar and Vidreres, alt. $100 \mathrm{~m}$; scrub with Quercus suber L., Phillyrea angustifolia L., Arbutus unedo L., Viburnum tinus L., Erica multiflora L., Erica scoparia L., Calluna vulgaris (L.), Ulex parviflorus Pourret, Cistus salviaefolius L., Cistus monspeliensis L., Daphne gnidium L., etc.; collected with a net by Nayrolles on unselected plants of the herbaceous stratum (station GE11); 4-IX-1987; 4 juvs.; 3 mounted.

Spain, prov. Gerona, near Banyoles, alt. $180 \mathrm{~m}$; garrigue with a lot of gramineae and Genista scorpius De Candolle, Erica multiflora L., Pinus halepensis Miller, Euphorbia nicaeensis Allioni, Dorycnium pentaphyllum Scopoli, Rosmarinus officinalis L., Catananche caerulea L., Phillyrea angustifolia L., etc., collected with a net on unselected plants by Nayrolles (station GE23); 17-XII-1987; 48 specimens; 13 juvs. and 7 ads. mounted.

Spain, prov. Tarragona, between Reus and L'Aleixar, about
$10 \mathrm{~km}$ from L'Aleixar, alt. $250 \mathrm{~m}$; scrub with Anthyllis cytisoides L., Ceratonia siliqua L., Pinus halepensis Miller, Cistus albidus L., Thymus vulgaris L., Ruta sp., Euphorbia characias L., Asparagus acutifolius L., Brachypodium ramosum Rœmer \& Schultes, Andropogon hirtus L., etc.; collected with a net by Nayrolles on unselected plants of the herbaceous stratum (station TA27); 14-IV-1988; 4 juvs. and $10 ; 3$ juvs. mounted.

Description. - Number of juv. st. $=4$

Size ad. male: $1.5-1.65 \mathrm{~mm}$; female: $1.65-$ $2.1 \mathrm{~mm}$.

Color. Very characteristic. Black head, and along back of body a black band with a narrow white stripe expanding into its anterior half. On posterior part of body, next to black band, one pair of purely white stripes composed of uric crystals. Flanks of old specimens brown colored, with rusty or greenish shade. This coloring can darken up to black. In this case, the specimens are completely black, except for the anterior mediodorsal narrow white stripe and both posterolateral white stripes. Ant. I dark, ant. II and III white, ant. IV very light ochre. Eyepatches black. Legs and furcula white.

Great abd. Trichobothria: A, B, C appearing at 2nd st., in a very open inverted pattern. Dorsal setae: macrochaetae.

Antennae (Figs. 10-15). Antennal ratios: in female, ant. I : II : III : IV = $1: 2.3: 3.2: 9.0$ and ant. : ceph. diag. $=2.3$; in male, ant. I : II : III : IV = $1: 2.4: 3.4: 9.7$ and ant $:$ ceph. diag. $=2.6$. Chaetotaxic variables: for $G: m=198.6 / s=4.88 /$ $\min =187 / \max =206$; for $I S h^{*}: m=0.53 / s=$ $0.030 / \min =0.48 / \max =0.60$; for $R S h: m=$ $0.49 / s=0.025 / \mathrm{min}=0.44 / \max =0.54$. Ant. II with 3 short and thin setae on large base, ant. III with 5 macrochaetae.

Head. Eyes: $8+8$; eyepatch with 2 setae. Dorsal part of head with large rough macrochaetae. One pair of postantennal setae. Labral formula: 6/5-5-4.

Legs. (CX1)il absent. Femur: cup present. Tibiotarsal inner side with spine-like and rather thick rough setae. Claws of all legs with a tunica and distinct pseudonychia. Tunica and pseudonychia grow

Figs. 10-15. Spatulosminthurus lesnei: 10, juv. 2nd st., ant. III, anterior view; 11, juv. 3rd st., ant. III, anterior view; 12, ad., ant. III, anterior view; 13-15, schematic representation of Figs. 10-12, same legend as in Fig. 4.

Figs. 16-17. Spatulosminthurus betschi ad.: 16, ant. III, anterior view; 17, schematic representation of Fig. 16, same legend as in Fig. 4. 
Table III. Appendicular chaetotaxy of Spatulosminthurus lesnei.

\begin{tabular}{|c|c|c|c|c|c|c|c|c|c|c|c|}
\hline \multirow{3}{*}{$\frac{A P}{A D}$} & $\Delta$ & \multicolumn{10}{|c|}{-} \\
\hline & $\Pi$ & \multicolumn{10}{|c|}{-} \\
\hline & $\Delta$ & \multicolumn{10}{|c|}{$\begin{array}{l}\text { D: io } T: \theta+1, a+1, i+1, p+1 \quad Q: i+2 \\
\text { io, } i+1, \text { and } i+2 \text { are small and slender setae on large base. }\end{array}$} \\
\hline \multirow[t]{5}{*}{ AT } & $\Pi$ & \multicolumn{10}{|c|}{ - } \\
\hline & \multirow[t]{4}{*}{$\Delta$} & & & $\mathbf{G e}_{0}$ & Gae & $\mathbf{G a}$ & Gai & Gi & Gpi & \begin{tabular}{|l|l|} 
Gp & $\mathbf{G p e}$ \\
\end{tabular} & \multirow{4}{*}{$\begin{array}{l}\text { Be, Bae, Ba, Bai, and Bp become macrochaetae during } \\
\text { devolopment. }\end{array}$} \\
\hline & & & Setae -1 & & 0 & & $\mathbf{T}$ & $\overline{0}$ & $T$ & 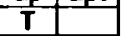 & \\
\hline & & & Setae 0 & $\mathbf{Q}$ & D & $\mathbf{D}$ & $\mathbf{Q}$ & $\bar{D}$ & D & D & \\
\hline & & & Setae +1 & & $T$ & $T$ & $\mathbf{Q}$ & & $\mathbf{Q}$ & $\mathbf{T}$ & \\
\hline \multirow[t]{6}{*}{$\overline{\mathrm{AQ}}$} & str & \multicolumn{10}{|c|}{$\begin{array}{l}\text { B completely zoned. Section M ouwhorled with } 19 \text { to } 21 \text { whorls. Right ouwhorlation. } \\
\text { Subsegmentation formula: } 1+20 / 22+1=(A)+(M 1,19 / 21+B A)+(B M+B B) \text {. Right subsegmentation. }\end{array}$} \\
\hline & \multirow[t]{3}{*}{$\bar{A}$} & $\Pi$ & \multicolumn{9}{|c|}{ Allpi } \\
\hline & & $\mathbf{H}$ & \multicolumn{9}{|c|}{ Alliea, Alllppe } \\
\hline & & $\Delta$ & \multicolumn{9}{|c|}{$\begin{array}{l}\text { Apical bulb: joined to the apex } \\
\text { Allpe is a blunt microchaeta. }\end{array}$} \\
\hline & \multirow[t]{2}{*}{$\begin{array}{l}\text { M } \\
\& \\
B\end{array}$} & $\bar{H}$ & \multicolumn{9}{|c|}{$\begin{array}{l}\text { Heae does not reach BA. For } \mathrm{Hea \theta}^{*}: m=10.50 ; \min =8 ; \max =12 \text {. } \\
\text { Hppe does not reach BA. For } H p p \theta^{\circ}: m=11.78 ; \min =10 ; \max =13 \text {. } \\
\text { For } h^{*}: m=11.1 ; \min =10.0 ; \max =12.0 \text {. The last } 5 \text { whorls of } M \text { without } H \text { setae. }\end{array}$} \\
\hline & & $\mathbf{G}$ & \multicolumn{9}{|c|}{$\begin{array}{l}\text { Setae of BB: P: BBe, BBpe Q: BBae, BBp / BA with several variable setae / } n B 0 \%(B M)=5-9 / n 80 \%(B)= \\
14-19 \text { / the last whorl on } M \text { with sometimes one or several absent setae / } n 80 \%(G)=192-204 \text {. }\end{array}$} \\
\hline
\end{tabular}

\begin{tabular}{|c|c|c|c|c|}
\hline \multirow[t]{2}{*}{$S B$} & pre & 二 & + & + \\
\hline & $\Delta$ & $\overline{P: 1}$ seta & P: 1 seta & $T: 1$ ov. org. \\
\hline SA & $\bar{\Delta}$ & - & $P: 1$ seta & P: I seta \\
\hline$c x$ & $\frac{\omega}{\Delta}$ & - & $\mathrm{P}: \mathrm{a \theta}, \mathrm{i1}, \mathrm{ms} \quad \mathrm{T}: \mathrm{Oi1}$ & P: ae, i1, ms T: a, Oi1 \\
\hline \multirow[t]{2}{*}{$\overline{T R}$} & $\Pi$ & - & - & $=$ \\
\hline & $\Delta$ & Q:pi & T:a2, Oi1, Oi2 Q: ae & T: a2, Oi1, Oi2 \\
\hline \multirow[t]{2}{*}{ FE } & $\Pi$ & - & - & pe1 \\
\hline & $\Delta$ & $\begin{array}{c}\text { delayed primordial seta: Q: ae3 } \\
\text { T: pe2, pe3 (T)Q: } p 2(o c=0.4) \\
\text { Q: a2, pi2, Op }\end{array}$ & T: a5, ai2, pe2 Q: a2, ai5, pe3, Op & $\begin{array}{c}\mathrm{T}: \mathrm{a} 5, \text { ai2 } \\
\mathrm{Q}: \mathrm{a2}, \mathrm{ai3}, \mathrm{ai} 4, \mathrm{ai5}, \mathrm{p} \in 2, \mathrm{p}, 3, \mathrm{Op}\end{array}$ \\
\hline \multirow[t]{4}{*}{$\pi$} & $\bar{v}$ & - & $\overline{V_{p}}$ & $V_{p}$ \\
\hline & $\bar{K}$ & - & 二 & \multirow{2}{*}{$\frac{1}{1}$} \\
\hline & FP & + & + & \\
\hline & $\bar{\Delta}$ & $\begin{array}{l}\text { T: 4ai1, 4pi1, Vai, Vpi, FSa, O1pe, } \\
\text { O2pe, O3pe, O4pe } \\
\text { (T):FSpe } \downarrow \text { (oc }-0.3) \\
\text { Q: } 2 a e, 3 a e, 3 a, 3 p, 4 a e 1,4 a 1,4 p 1, \\
\text { 4ai2, 4pi2, FSo } \uparrow, \text { FSai, FSpi, O1ae } \\
\text { le and lpe are spatulate setae. } \\
\text { Vi is a microchaeta on large base. }\end{array}$ & $\begin{array}{l}\text { T: 4ai1, 4i1, Vai, Vpi, FSa, O1pe, } \\
\text { O2pe, O3pe, O4pe } \\
\text { (T)O:FSpe }(0 c=0.4) \\
\text { Q: 2ae, 3ae, 3a, 3p, 4ae1, 4a1, 4pi1, } \\
\text { 4p1, 4ai2, 4pi2, FSeT, FSai, FSpi, } \\
\text { O1 ae } \\
\text { le and lpe are spatulate setae. } \\
\text { Vi is a microchaeta on large base. }\end{array}$ & $\begin{array}{l}\text { T: 3ai, 3i, 4ai1, 4i1, Vai, Vpi, FSa, } \\
\text { O1pe, O2pe, O3pe, O4pe } \\
\text { Q: 2ae, 2a, 2p, 3ae, 3a, 3pi, 3p, 4ae1, } \\
\text { 4a1, 4pi1, 4ai2, 4pi2, FSeT, FSai, } \\
\text { FSpi, FSpe } \downarrow \text {, O1ae } \\
\text { lo and lpe are spatulate setae. } \\
\text { Vi is a microchaeta on large base. }\end{array}$ \\
\hline
\end{tabular}

\begin{tabular}{|c|c|c|c|c|c|c|c|c|c|c|c|c|c|}
\hline MA & $\Pi$ & & & & & & & & & $=$ & & & \\
\hline & $\Delta$ & & & & & & & T: $\mathbf{a}$ & pe2 & & : $p \theta 3$ & $(\alpha=0.7)$ & \\
\hline DE & $\Delta$ & & & $G_{\theta}$ & Gao & $\mathrm{Ga}_{\mathbf{a}}$ & Gai & Gi & Goi & Go & Goo & Other setae: $\quad P: 1 p$ & Q: 3p, 4p, 5p, BBpi \\
\hline & & & $T$ & $P$ & $P$ & $P$ & $P$ & $\bar{P}$ & $P$ & $p$ & $P$ & $\alpha c(I V p i)=0.4$ & \\
\hline & & & $\|$ & & $\mathbf{P}$ & $\mathbf{P}$ & $\mathbf{P}$ & & $\mathbf{P}$ & $\mathbf{P}$ & $\bar{P}$ & & \\
\hline & & Whorls & iil & & $\mathbf{P}$ & $\mathbf{P}$ & $\mathbf{P}$ & & $\mathbf{P}$ & $\mathbf{P}$ & $\bar{P}$ & & \\
\hline & & I to VI & IV & & $\mathbf{P}$ & $\mathbf{P}$ & & & (T)a & $\mathbf{P}$ & $\mathbf{P}$ & & \\
\hline & & & $\mathbf{V}$ & & $\mathbf{P}$ & $\mathbf{P}$ & & & $\mathbf{T}$ & $\mathbf{P}$ & $\mathbf{T}$ & & \\
\hline & & & VI & & & $\mathbf{P}$ & & & $\mathbf{Q}$ & 0 & $\bar{T}$ & & \\
\hline & & WhorlB & & $\mathbf{T}$ & & $\mathbf{P}$ & & & $\mathbf{P}$ & $\mathbf{P}$ & $\overline{\mathbf{P}}$ & & \\
\hline MU & $\Delta$ & $\begin{array}{l}\text { y: D } \\
\text { me sli }\end{array}$ & mu & & & & & & & & & 1st instar the oute & \\
\hline
\end{tabular}


Table IV. Appendicular chaetotaxy of Spatulosminthurus betschi.

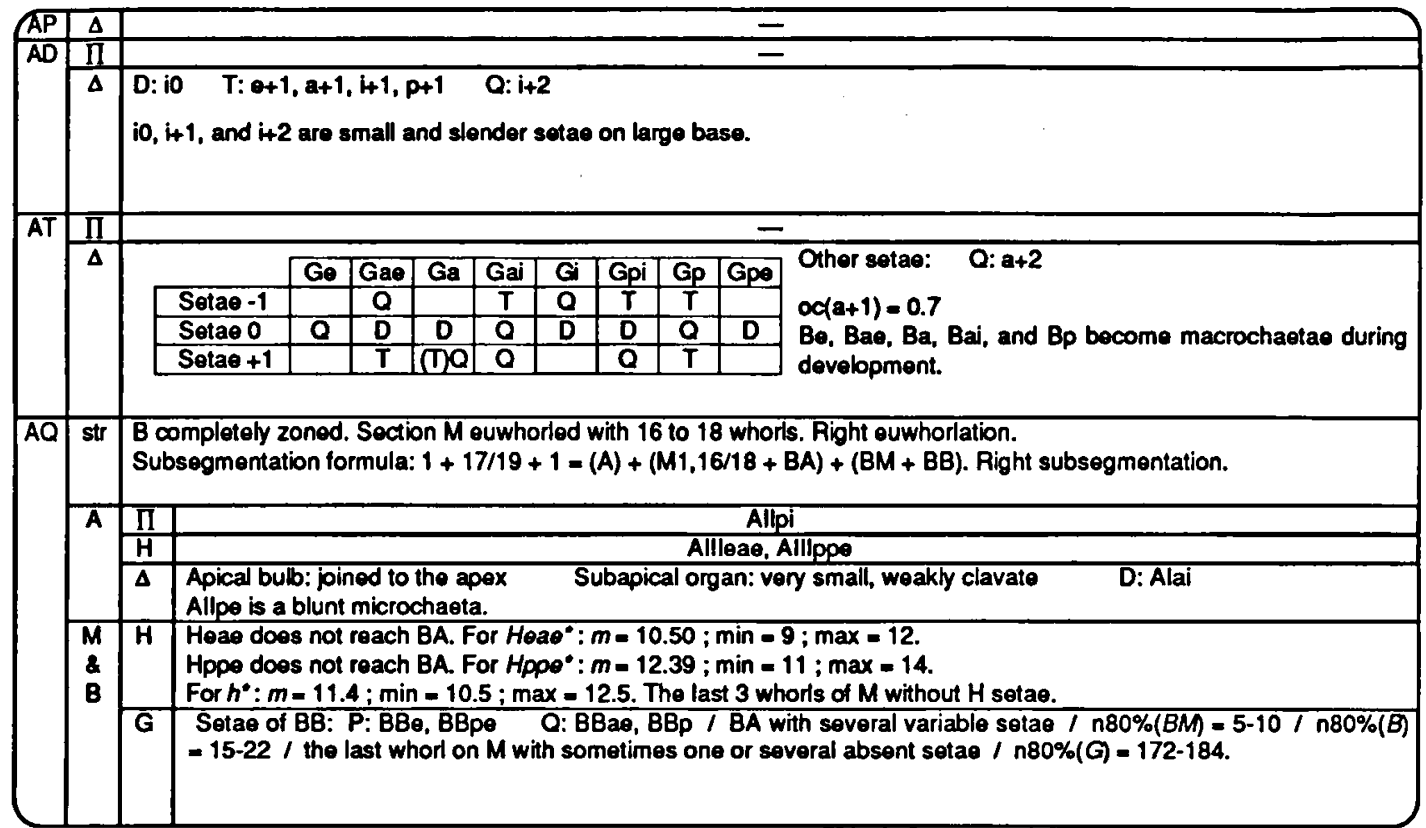

\begin{tabular}{|c|c|c|c|c|}
\hline \multirow{2}{*}{$\mathrm{SB}$} & pre & 二 & \multirow{2}{*}{$\frac{+}{P: 1 \text { seta }}$} & + \\
\hline & $\Delta$ & P:1 sota & & P: 1 seta $\quad \mathrm{T}: 1$ ov. org. \\
\hline SA & $\Delta$ & - & P:1 seta & P:1 seta \\
\hline $\mathrm{CX}$ & $\Delta$ & $\overline{-}$ & P: ao, i1, ms T: Oi1 & $P: a \theta, i 1, m s \quad T: a$, Oi1 \\
\hline \multirow[t]{2}{*}{ TR } & $\bar{\Pi}$ & $\overline{-}$ & - & - \\
\hline & $\Delta$ & Q:pi & $\mathrm{T}: 22, \mathrm{Oi1}, \mathrm{Oi2}$ & T: a2, Oi1, Oi2 \\
\hline \multirow[t]{2}{*}{ FE } & II & - & $\overline{-}$ & $p+1$ \\
\hline & $\Delta$ & $\begin{array}{l}\text { delayed primordial seta: } Q: a \theta 3 \\
\begin{array}{ll}\text { T: pi2, pe2, pe3 } & \text { Q: a2, p2, Op }\end{array}\end{array}$ & $\mathrm{T}: \mathrm{a5}, \mathrm{ai} 2, \mathrm{pe2} \quad \mathrm{Q}: \mathrm{a2}, \mathrm{ai5}, \mathrm{p} \theta 3, \mathrm{Op}$ & $\begin{array}{c}\mathrm{T}: \mathrm{a} 5, \text { ai2 } \\
\mathrm{Q}: 22, \text { ai3, ai4, ai5, pe2, po3, op }\end{array}$ \\
\hline \multirow[t]{5}{*}{$\pi$} & \multirow{3}{*}{$\begin{array}{l}\bar{V} \\
K \\
F P\end{array}$} & $\overline{-}$ & $V_{p}$ & $\overline{V_{p}}$ \\
\hline & & $=$ & 二 & $=$ \\
\hline & & + & + & + \\
\hline & \multirow[t]{2}{*}{$\Delta$} & 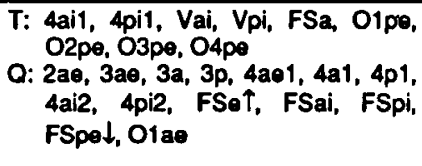 & $\begin{array}{l}\text { T: } 4 a 11,411, \text { Vai, Vpi, FSa, O1pe, } \\
\text { O2pe, O3pe, O4pe } \\
\text { Q: } 2 a \theta, 3 a e, 3 a, 3 p, 4 a e 1,4 a 1,4 p i 1, \\
\text { 4p1, 4ai2, 4pi2, FSe } \uparrow, \text { FSai, FSpi, } \\
\text { FSpo } \downarrow, \text { O1ae }\end{array}$ & \multirow{2}{*}{$\begin{array}{l}\text { T: 3ai, 3i, 4ai1, 4i1, Vai, Vpi, FSa, } \\
\text { O1pe, O2pe, O3pe, O4pe } \\
\text { Q: } 2 a \theta, 2 a, 2 p, 3 a \theta, 3 a, 3 p i, 3 p, 4 a \theta 1, \\
\text { 4a1, 4pi1, 4p1, 4ai2, 4pi2, FSeT, } \\
\text { FSai, FSpi, FSpe } \downarrow \text {, O1ae } \\
\text { le and lpe are spatulate setae. } \\
\text { Vi is a microchaeta on large base. }\end{array}$} \\
\hline & & $\begin{array}{l}\text { le and lpe are spatulate setae. } \\
\text { Vi is a microchaeta on large base. }\end{array}$ & $\begin{array}{l}l e \text { and lpe are spatulate setae. } \\
V_{i} \text { is a microchaeta on large base. }\end{array}$ & \\
\hline
\end{tabular}

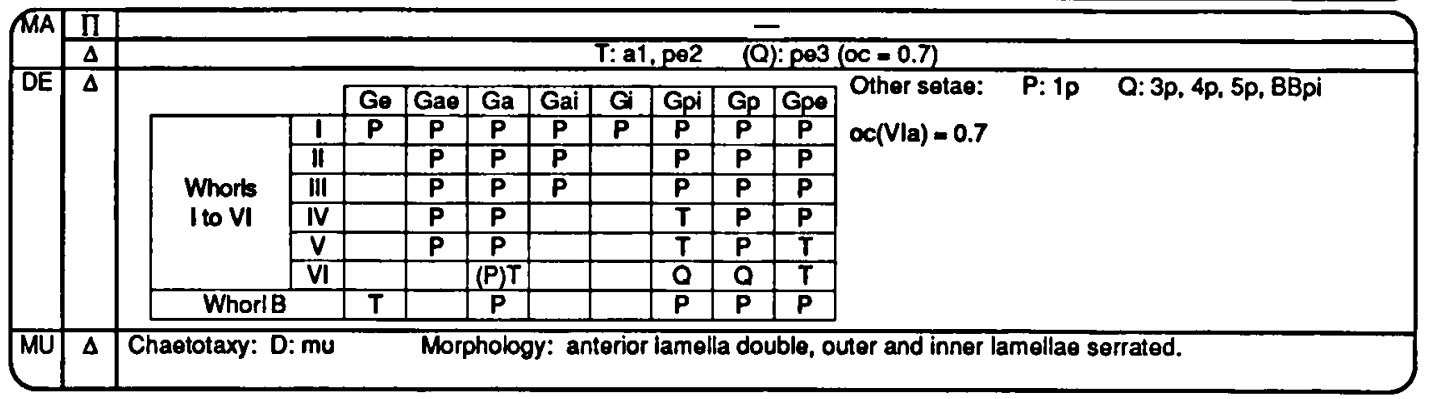


during development. Tooth on inner side of claw on mesolegs and hindlegs undergoes a great decrease in size; in adult this tooth remains distinct on forelegs, but becomes minute on mesolegs and minute or absent on hindlegs. Outer lamella of empodial appendage double and smooth on forelegs, double and serrated on meso- and hindlegs. Empodial filament long on forelegs, very short on meso- and hindlegs.

Ventral tube. Sacs warty from 2 nd st. on. Chaetotaxy: apical flaps with one pair of primary setae. One pair of setae on corpus appears on 4th st.

Retinaculum. Rami bidentate from 1st st. on (apical tooth distinct and joined with median tooth at 1st st., and becoming indistinct during development). Chaetotaxy: on anterior lobe one pair of setae appears at 3rd st., another pair at 4th.

Furcula. Cf. chaetotaxic table.

Small abd. Made up of abd. V + abd. VI. Trichobothrium: D present. Genital papilla of male medium-sized with $26-29$ setae. In female the lateral circumanal setae on upper valve with a twisted blade. An. app. short and broad, its inner edge thick and pointed, and its outer edge being a finely serrated lamella. Ratio an. app. : mucro $=0.3$.

Spatulosminthurus betschi Nayrolles, 1990 (Figs. 16-17, Table IV)

In my previous paper (Nayrolles, 1990) the antennal chaetotaxy was not studied. The description of this species is here completed by giving the appendicular chaetotaxic table as well as adding the drawing of the chaetotaxy of ant. III (Figs. 16-17) and the following information about the chaetotaxic variables of ant. IV: for $G: m=178.7 / s=4.36 / \mathrm{min}=$ $170 / \max =188$; for $I S h^{*}: m=0.63 / s=$ $0.024 / \min =0.58 / \max =0.67 ;$ for $R S h: m=$
$0.56 / s=0.023 / \min =0.51 / \max =0.60$. Ant . II with 3 short and thin setae on large base, ant. III with 5 macrochaetae.

Discussion. - In my description of S. betschi, I gave the following characters for distinguishing $S$. lesnei from $S$. betschi: the color pattern, the number of subsegments on ant. IV, and the inner tooth of the claws. From the fourth instar on, the absence of (CX2)a, (TI3)4pl, (AT)p0, and (AT)a +2 in S. lesnei only, also permit us to readily distinguish both species.

\section{References}

Altner, H., K.D. Ernst \& G. Karuhize, 1970. Untersuchungen am Postantennalorgan der Collembolen (Apterygota). I. Die Feinstruktur der postantennalen Sinnesborste von Sminthurus fuscus (L.). Z. Zellforsch., 111: 263-285.

Betsch, J.-M. \& M.-C. Betsch-Pinot, 1984. Contribution à l'étude des Sminthurus (Collembola, Symphypleona). Annls. Soc. r. zool. Belg., 114 (1): 71-81.

Betsch, J.-M. \& A. Waller (in press). Schematic chaetotaxy on head, thoracal, and abdominal segments in Symphypleona (Collembola, Insecta). Acta zool. Fenn.

Ellis, W.N., 1973. Allacma fusca (L.) and Allacma gallica (Carl) in Holland (Collembola: Sminthuridae). Ent. Ber., 33: 170179.

Nayrolles, P., 1990. Description d'une nouvelle espèce de Spatulosminthurus (Collemboles Symphypléones). Bull. Soc. Hist. nat. Toulouse, 126: 65-71.

Nayrolles, P., 1993. A standardized description of European Sminthuridae (Collembola, Symphypleona), 1: genera Lipothrix, Gisinurus, and Caprainea. Bijdr. Dierk., 63 (1): 43-60.

Yosii, R. \& C. Lee, 1963. On some Collembola of Korea with notes on the genus Ptenothrix. Contr. biol. Lab. Kyoto Univ., 15: 1-37.

Received: 26 July 1993

Revised: 24 December 1993 